\title{
Soldier safety and performance through wearable devices
}

Richard Murdock, Joshua Hagen

Richard C. Murdock, Joshua A. Hagen, "Soldier safety and performance through wearable devices," Proc. SPIE 10639, Micro- and Nanotechnology Sensors, Systems, and Applications X, 106391A (8 May 2018); doi: 10.1117/12.2304899

SPIE. Event: SPIE Defense + Security, 2018, Orlando, FL, United States 


\title{
Soldier Safety and Performance through Wearable Devices
}

\author{
Richard C. Murdock*a and Joshua A. Hagen ${ }^{\mathrm{b}}$ \\ a Airman Systems Directorate, 711th Human Performance Wing, Air Force Research Laboratory, \\ Wright-Patterson AFB, OH 45433; ${ }^{\mathrm{b}}$ Human Performance Innovation Center, Rockefeller \\ Neuroscience Institute, West Virginia University, Morgantown, WV
}

\begin{abstract}
Wearable technologies have the ability to change how we perceive, and make decisions about, our health and well-being. In the military, utilizing these emerging technologies in training or operations offers potential life-saving and performance enhancement benefits. Up until now, very limited physiological data collection has been performed due to the overall integration, form-factor, power limitations, and data feedback to the user from wearable monitoring devices. The explosion of the wearables sector in the commercial arena has pushed industry to solve a lot of these issues for the consumer market, allowing for new monitoring opportunities within the military as well. This manuscript discusses a couple of the use cases for wearable technologies within military environments, specifically heat stress injury prevention and performance monitoring during training. Additionally, some preliminary wearable device gold-standard testing is discussed. From the applications described, it can be seen how integration of these technologies has allowed for safer training environments, but also has improved training effectiveness and sustained performance enhancement.
\end{abstract}

Keywords: Wearable technology, physiological performance, heat stress, biomarkers

\section{INTRODUCTION}

Currently, we have over 1700 sensors on high performance aircraft providing real-time feedback about hundreds of airframe parameters to monitor its performance and health status, yet only recently have we put the first sensor on the human who is piloting these multi-million dollar assets to monitor their performance and health status. This lack of physiological monitoring feedback holds true for every other Airmen, who perform their missions with the only health and wellness checks and Physical Fitness Testing (PFT), both occurring every 12 months, and is not linked in any way to the actual performance of their duties, training, or mission. The recent explosion of commercially available physiological monitoring technologies is therefore of significant interest to the military to help fill these human performance monitoring gaps. These technologies include wrist worn wearable devices such as various Fitbits, Garmins, Apple, and Sumsung devices, as well as strap-based devices such as certain Polar and Garmin products, alongside more research oriented devices such as Zephyr and Hidalgo Equivital.

For military applications, the primary challenge lies within the dilemma of using commercial-of-the-shelf (COTS) devices, which might provide an $80 \%$ solution at a cost effective price-point; however, when attempting to work with the commercial company to modify a mass manufactured device to be a $100 \%$ solution, there is little to no interest. The alternative is for the Department of Defense (DoD) to initiate a solution, which may take years to develop, test, and manufacture to a high technology readiness level (TRL), only to have it be obsolete to the customer by that time. Additionally, each of these technologies has associated software for the user, but they are specific only to the device's data stream, which is less than ideal, and the data analytics are geared toward general consumer use. This means that there is very little ability to adapt or personalize the analytics to be more applicable to military settings. Recent press announcements from multi-billion dollar corporations such as Samsung, Apple, and Intel lead us to believe that an ideal form factor device that can measure all of the necessary metrics will become available sooner than later (Samsung, Intel, Apple). However, the lack of flexibility of the analytics and personalization of software will likely remain true. The goal of any wearable technology is to collect quantitative data, perform analysis on that data to indicate how it compares to the wearer's personal norm, the group norm, or against performance metrics, and then provide feedback to the user to implement a prescriptive or corrective action. A significant challenge for the commercial industry of wearable sensors is data analysis and reporting to the user useful metrics upon which to act. In many cases, a single sensor contains more information than the user can consume, and also make sense of what information is important to base any change in behavior off of. For the military, being able to fuse all of the data streams, using wearable sensors and/or other data sources, into actionable recommendations as to reduce overtraining, increase readiness, and reduce injuries.

Micro- and Nanotechnology Sensors, Systems, and Applications X, edited by Thomas George,

Achyut K. Dutta, M. Saif Islam, Proc. of SPIE Vol. 10639, 106391A · @ 2018 SPIE

CCC code: $0277-786 \mathrm{X} / 18 / \$ 18 \cdot$ doi: $10.1117 / 12.2304899$ 


\section{RESULTS AND DISCUSSION}

There are many physical demands placed on the warfighters of the DoD. Every Airman is required to maintain minimum physical fitness standard to remain in the Air Force. Every Airman can benefit from a higher level of health and wellness. Especially relevant to this idea of a smartphone based trainer and tracker is the news that the Air Force will be shutting down Health and Wellness Centers AF wide in the next year ${ }^{1}$. Military operators perform at an elite level, and can benefit from technologies that can allow them to perform at an even higher level from personalized training, tracking, and recovery. Physical training is an integral part of the warfighter's daily performance, regardless of the mission space that they serve. Training effectively, getting the proper recovery and nutrition, and continuously being aware of your physical and cognitive workload can all lead to more productive and healthy lives; "What can be measured can be managed" (Drucker). By applying physiological monitoring technologies, being able to do all the things previously mentioned become possible. While there is an influx of commercial devices that address some of these issues, none of them are comprehensive in sensor metrics, and they have a very small amount of individualization, and are largely geared toward the average consumer and not the average military operator. With budget and manpower constraints on HP staff in the military, being able to train and track physical metrics remotely with a readily accessible software package becomes even more advantageous. Additionally, musculoskeletal injuries have a higher rate of occurrence with overtraining and inadequate recovery ${ }^{2}$. Knowing the real effort exerted by the trainee, the trainers can augment the training demands based on real recovery needs, potentially leading to a reduction in musculoskeletal injuries. Utilizing the proposed technologies will have a unique, personalized, and accurate way of measuring physical workload both cardiovascular and muscle fatigue. The objective with aggregating the performance data will be to develop predictive algorithms for personalized training and tracking to maximize the operational readiness and effectiveness of any operator.

\subsection{Use of Wearable Monitoring Technologies for Performance Monitoring}

Recent advances in microelectronics, biotechnology, and nanotechnology have enabled new paradigms in wearable monitoring. Today we can monitor cardiac waveforms in a shirt or strap, G-forces using a simple accelerometer, and can analyze your stress from heart-rate-variability. Currently, there are two primary categories of commercially available physiological monitors; 1) consumer-grade highly wearable devices, but extremely low fidelity data and ruggedness (e.g. FitBit) and 2) research-grade higher fidelity data devices, but very complex with poor usability and wearability ${ }^{3}$. The DoD overall is taking multiple approaches to find wearables that fit military applications. First is to identify consumer-grade devices that have accuracy within acceptable limitations. At the Air Force Research Laboratory (AFRL), using both existing and novel technologies, we are working towards a fully integrated solution with streamlined and easy to understand data for a complete picture of health, wellness, stress, and advanced human performance for both civilian and military applications all over the world. These technologies can significantly enhance the safety of military operations, the effectiveness of disaster relief, and improve provision of health care in remote areas with limited infrastructure. More recently, specific focus has been on the physiological performance monitoring during selection and training for these programs, as well as the ability to monitor and maintain a high level of physiological readiness for current team members. The need comes from the lack of knowledge among these customers on the proper wearable device selection for physiological monitoring and the overwhelming amount of data that can be obtained from each device used. Development has been focused on selecting the right device, or devices, for each customer depending on their specific requirements and use environment, help to digest the information from each device, and determine the key performance parameters for each customer to use to predict performance-related trends or changes. Essentially this comes down to a sense-assess-augment loop, as shown below in Figure 1. 


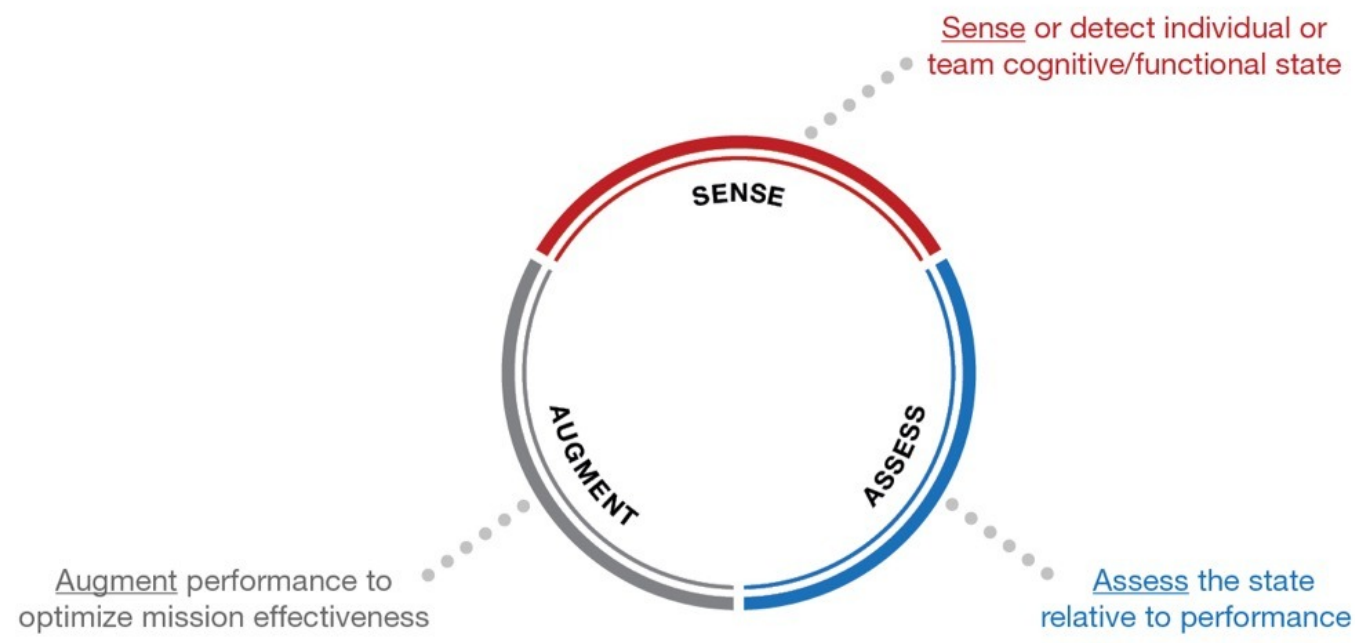

\section{Apply the right sensors and assessment methods to enable} the successful deployment of personalized augmentations

Figure 1. Sense-Assess-Augment Loop Diagram.

Selection of devices for the use case is usually a process of evaluating tradeoffs when attempting to use COTS devices. The usual parameters/features in question are measurement accuracy, wearability, ruggedness, general usability, battery life, ability to live stream physio data, and the ability to store/export data, and it's usually a combination of multiple of these, not just one, that dictates the proper device. To help educate our military user groups, AFRL took the initiative to start performing testing, evaluation, and validation of wearable technologies that might be of interest. Figure 2 below shows the results of these early trials. In short, fifteen healthy young adults consented for participation in the heart rate and caloric expenditure trials of this study with average age $32.1 \pm 7$, BMI $27.9 \pm 3.9$ and percentage body fat 20.9 \pm 7.8 (mean $\pm \mathrm{SD}$ ). Each participant wore between 3-5 wearable devices simultaneously at different locations on the body. The included devices were Polar RC3 (Polar), Zephyr BioHarness3 (Zephyr), Mio Fuse (Mio), Fitbit Surge (Surge), Garmin Fēnix3 (Garmin), Suunto Ambit3 Peak (Suunto), Fitbit Charge (Charge), Adidas SmartFit (SmartFit), and Apple iWatch (iWatch), Under Armour/JBL Earbuds (JBL), and Jabra Elite Sport Earbuds (Jabra). Three of the eleven wearable devices were chest worn (Polar, Zephyr, and Suunto), two were in-ear (JBL, Jabra), and the other eight devices were wrist worn. Polar was used as the reference system for heart rate trials given its previous validation studies ${ }^{4-9}$. 

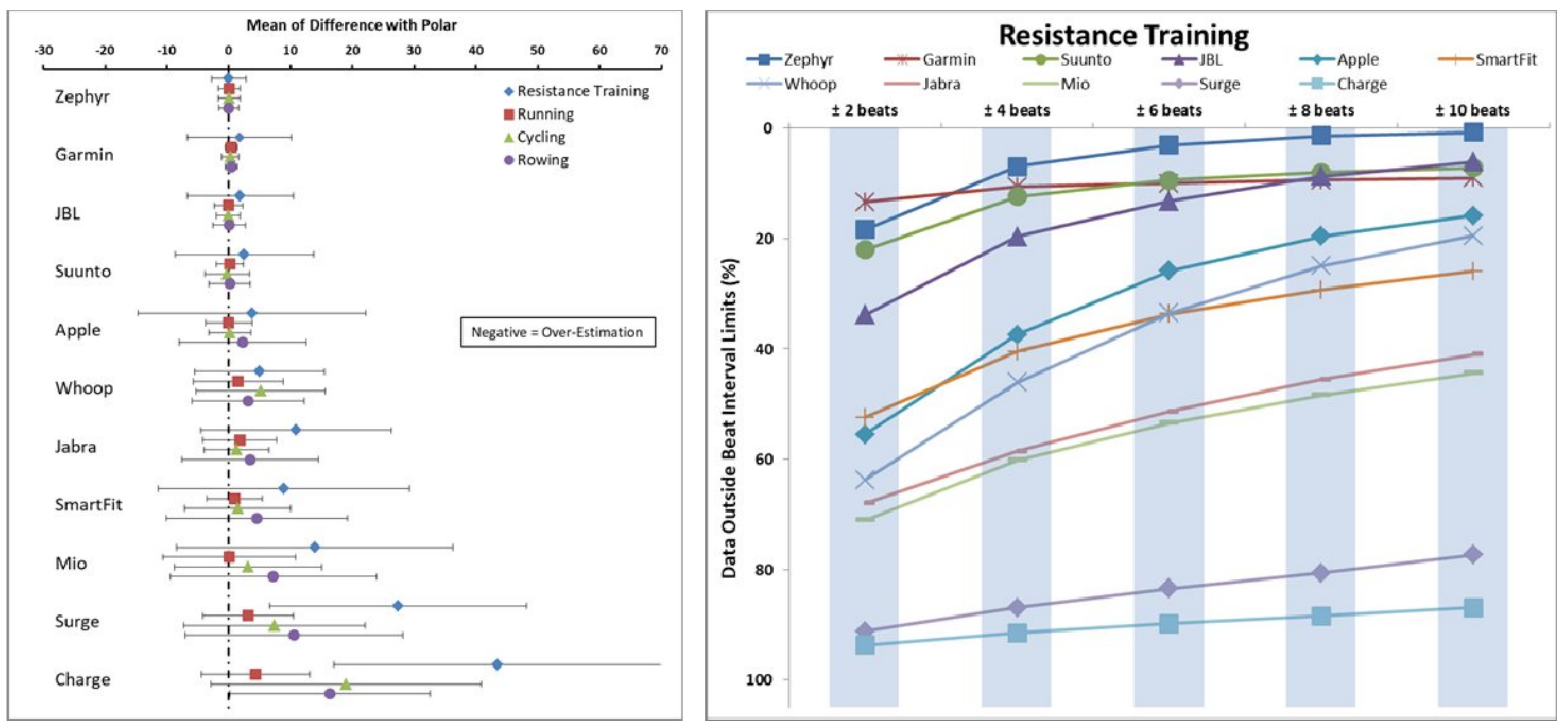

Figure 2. (Left) Comparison of mean of difference between Polar and various evaluated devices for heart rate during resistance training, running, cycling, and rowing. (Right) Percentage number of outliers for different beat intervals during resistance training trials.

Comparison of group averages were tabulated for heart rate against the Polar device. It should be noted that a mere comparison of group means does not reflect the reliability of a device and that the difference of values between each wearable device and its reference device gives a more realistic comparison. This data can be seen for heart rate Figure 1 (left). Additionally, if a device has fixed bias, the values of the wearable devices can be adjusted to match the reference system; however, limits of agreement should be used to determine if the devices can be used interchangeably.

During resistance training trials, all of the devices displayed a significant fixed bias and a significant proportional bias for heart rate data. However, the limits of agreement of the heart rate data were minimal for Zephyr. During running trials, 6 of the 7 devices displayed a significant fixed bias, and 6 of 7 devices displayed a significant proportional bias for heart rate. Mio did not show a significant fixed bias, and SmartFit did not show a significant proportional bias during the running trials. The limits of agreement for this trial were minimal for Garmin. During cycling trials, all 7 devices displayed a significant fixed bias for heart rate, however only Zephyr, Mio, Garmin, and Suunto showed a significant proportional bias for heart rate. The limits of agreement for cycling were minimal for Garmin for heart rate. Finally, for heart rate data during rowing trails, significant proportional bias was present in all of the devices, while significant fixed bias was displayed in 6 of 7 devices. Zephyr was the only device that did not display a significant fixed bias for heart rate during rowing trials.

There are multiple things this data demonstrates, with the most important being that chest strap devices are less affected by the type of activity than wrist worn or even in-ear devices. Wrist worn photoplethysmography (PPG)-based devices are more prone to measurement error due to changes in blood flow around the wrist arear from wrist flexion or extension, excessive or rapid arm movements, and also from tightly grasping an object. Chest strap devices in the upper torso appear to stay in position better throughout a wide variety of movements. Another observation is that choice of device should be dictated first by primary type of activity being monitored, then by user comfort/compliance, and finally be acceptable device accuracy. For example, if the activity is primarily running, a wrist worn device may be acceptable as the accuracy is still within a reasonable limit, and user comfort/compliance will be higher than with a chest worn device. However, if the activity requires strenuous activity with an increased amount of variety of movements, such as in the resistance training example above, a chest strap device is the only adequate option as the wrist worn devices are still only within $+/-6 \mathrm{bpm}$ for $70 \%$ of the data (best case scenario, as seen with the Apple iWatch. Interestingly, some devices had large amounts of missing data (60-80\%), which it was concluded that it was due to adaptive data sampling rates in the device to either conserve battery or memory.

Once devices are chosen, collection of performance data over time, in conjunction with quantitative performance metrics, is needed to start to combine various data streams and develop predictive performance models. In athletics, the 
quantitative performance data can come from game stats and sport positional metrics (i.e. 40-yd dash times). For the military, this is much more difficult to define and is an on-going problem attempting to be solved as different units have different roles and thus different performance targets or standards. As discussed previously, the objective with aggregating the performance data will be to develop predictive algorithms for personalized training and tracking to maximize the operational readiness and effectiveness of any operator and provide real-time feedback to training cadre or command staff. A conceptual view of this interface can be seen below in Figure 3.
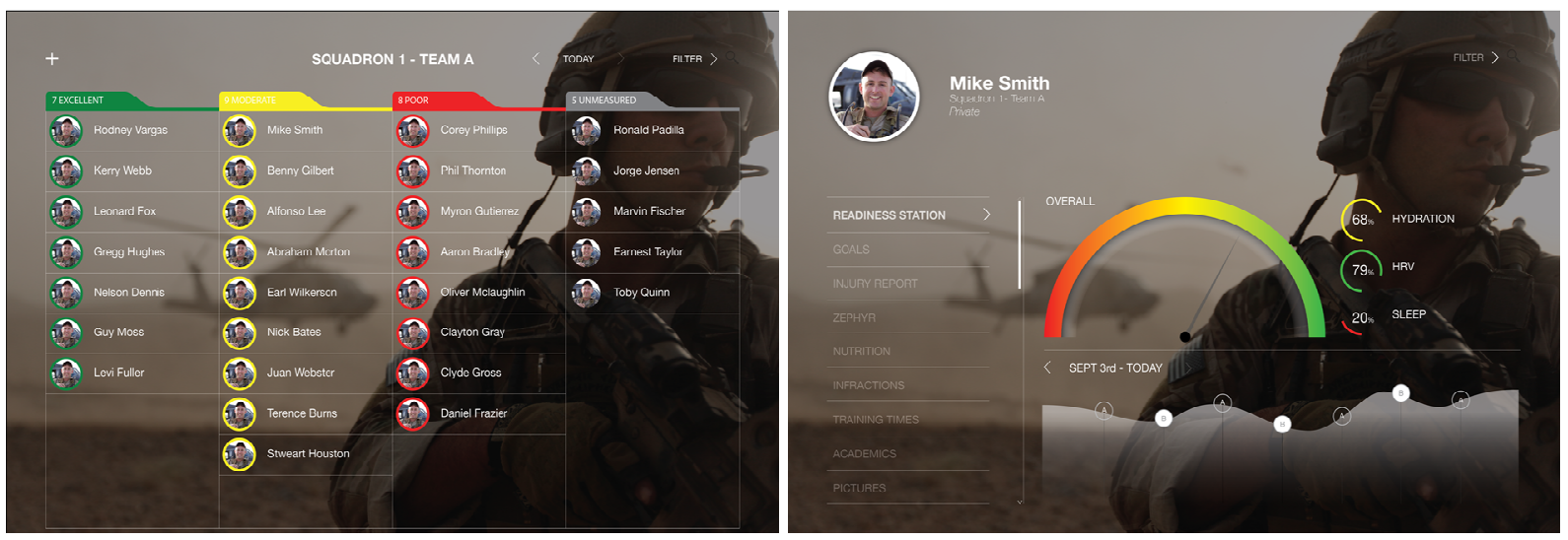

Figure 3. Conceptual interface for data aggregation and feedback system.

\subsection{Use of Wearable Technologies for Health and Safety Monitoring}

Statistics show that in a 3-year period, as much as 600 medical events occurred during the training pipeline ${ }^{10}$. Over a 14 year period (1977-2001), 126 non-traumatic sudden deaths occurred during U.S. basic military training ${ }^{11}$. Specifically, heat stroke is responsible for $2.5 \%$ of deaths in each branch of service including the Army, Navy, Marine Corps, and Air Force ${ }^{11}$. Wearable devices that provide real-time physiological changes due to exertion may aid in early identification of potential for heat stress illness/injury, which can prevent or mitigate a more serious event.

The U.S. Army Research Institute of Environmental Medicine (USARIEM) developed the Estimated Core Temperature, or ECTemp, algorithm which provides accurate estimates of core temperature simply by recording heart rate, allowing mission leaders to detect if a soldier is at risk of heat stress illness. The ECTemp algorithm uses minuteto-minute measures of heat rate to accurately estimate core body temperature. The algorithm was licensed to Zephyr Technology a few years ago to use as one of the features of its Bioharness, which would monitor team member health statuses to prevent and mitigate injury. Warfighters will go through hard training that can cause their body temperatures to increase and the ECTemp algorithm can provide mission leaders the situational awareness on who is getting hot and who should not be getting hot. Using information from the algorithm, military leaders can decide to slow the pace or remove some of the load during training activities. The algorithm has proven to be successful in real-world scenarios. In the past year, AFRL has used the Zephyr system, equipped with the ECTemp algorithm, to monitor trainees under the Battlefield Airman Training Group to help identify and mitigate over 30 cases of heat stress and prevent more serious heat stress casualty situations.

Presented here is the initial work to evaluate the effectiveness of such monitoring techniques, specifically using the Zephyr Bioharness 3 system (Figure 4), to minimize or eliminate heat stress events/casualties while training in hot environments. Twenty percent of basic trainees have been reported to experience a heat stress event during training. The current effort was put in place to test for an optimal system that will allow for real-time monitoring of trainee and operator vital signs during multiple military training events. 


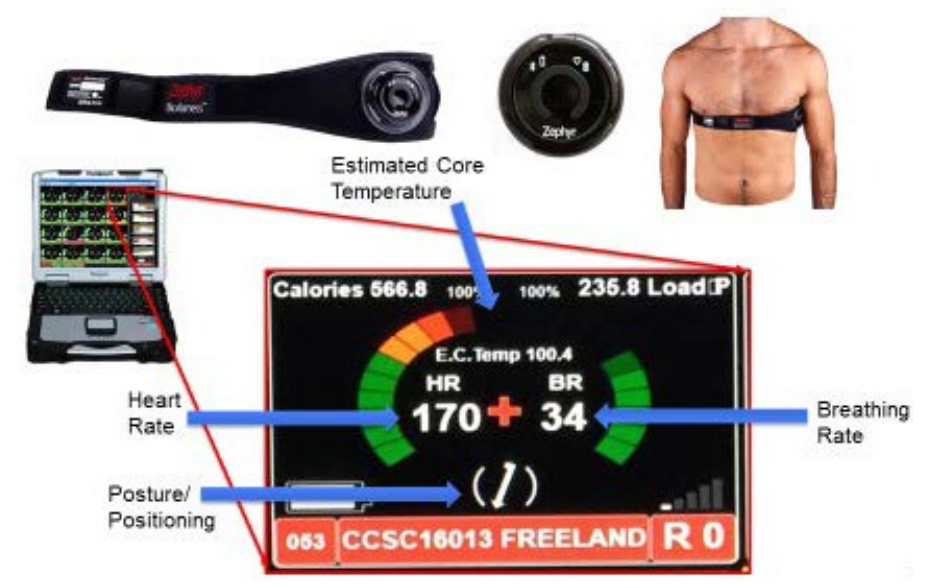

Figure 4. Zephyr BioHarness and System Functionality

Real-time physiological monitoring has the potential to reduce the number of heat stress events by alerting members of the cadre when trainee vital signs are out of range for an extended period of time. In addition, instances may occur where several trainees are experiencing high "estimated core temperatures" or heat rate levels that exceed $80 \%$ of one's maximum heart rate. Exercises that increase the likelihood of a heat stress event can be mitigated by embedding cooling techniques into the task (i.e., moving the event into an aquatic environment, adding cooling gear, increasing the number of breaks between bouts of exercise). The example below (Figure 5) led to a specific request for vital signs to be continuously monitored during high stress events. Heart rate data was collected during a military training course during a ruck march on extended training day (ETD). The red line represents this trainee's heart rate during the task $(\sim 3: 00-$ 4:12AM). At some point during this ruck march, this trainee's "beats per minute" rose above $80 \%$ of his maximum heart rate (blue line) and for longer than 20 minutes. Also shown on the bottom right is a comparison of the heart rate trends between another trainee in the course and this particular trainee. Following this extended increase, the trainee fell to the ground and was treated by the medical staff on site. It was determined that the trainee should not complete the event, thus, he was unable to continue the training course. This event motivated the Course Chief as well as the research team to establish performance and safety monitoring as a standard during such high stress events.

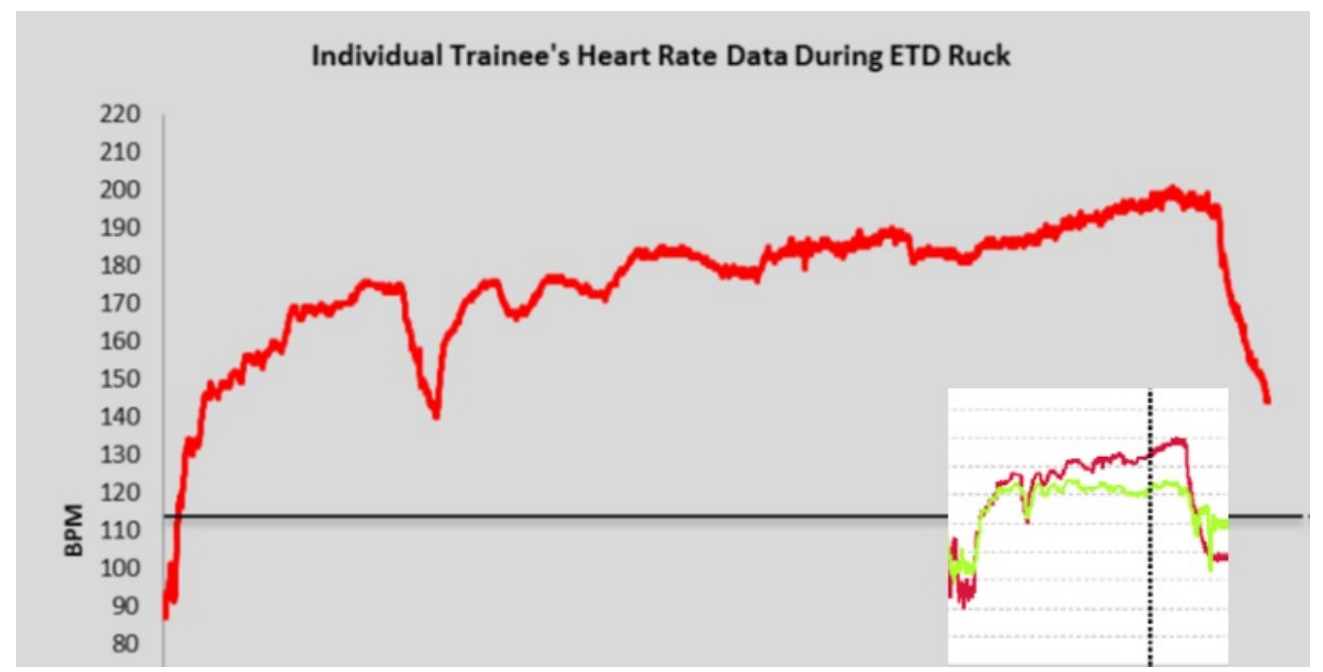

Figure 5. Zephyr BioHarness and System Functionality 
Over a one year time span, greater than 1,942 potential heat stress events were monitored during high stress events. During these events, 5 situations led to a near loss of conscious, thus was recorded as a missed event. However, high ECTs and/or irregular heart rate patterns were not signaled in these situations. Rather, exhaustion better exemplifies these specific cases. There was a total of 90 events that were mitigated and ranged from zero interference with training to the identification of vitals that needed checked and ended with the trainee unable to complete the task (there is no indication of return vs. no return to the course). Of these events, $77.89 \%$ led to the trainee either staying in training or returning back to training once a mitigation was in place such as hydration or movement into a cooler environment. This $77.89 \%$ was estimated to save the Air Force $\sim \$ 1 \mathrm{M}$ in possible washback trainee costs. Moreover, $16.84 \%$ of events led to further assessment during training and a medical decision to send the trainee to medical for further evaluation. Though we were unable to track if each of these events led to a return to training or a washback into a later course, the medical evaluation may have alleviated a more serious health issue that may have otherwise been unidentified.

\section{CONCLUSIONS}

The technologies discussed in this manuscript have the potential to alter the way physical training, recovery, and readiness are managed. This could potentially lead to musculoskeletal injury reduction, decreased physical training costs, improved retention by improving PFT scoring. While all of these concepts have benefits beyond the military, the specific beneficiary in the military of this efforts are the operators themselves. The technologies will be custom designed for their mission space for the purpose of improved physical and cognitive performance, improved situational awareness of team readiness, potential to reduce injuries through real-world recovery predictions, and amongst numerous other benefits. Future technical challenges that are faced with wearable devices are: high wearability, high durability/ruggedness, extended battery life, and secure data communication/transfer methods. Specific data analytics/algorithm challenges are: large data sets, varying time scales of physiological data, model development with limited replicates (individuals), and relating the physiological data to real-world performance. Additionally, in the future, incorporation of real-time biomarker detection platforms will further enhance the predictive power of these algorithms. These devices will need to be low-cost, expendable or reusable, highly selective to the analyte, have high sensitivity/accuracy, and be minimally invasive to the user.

\section{REFERENCES}

[1] [AF Times]: http://www.airforcetimes.com/article/20140604/BENEFITS07/306040069/Health-wellness-centersclose

[2] Kibler WB, Chandler TJ, Stracener ES, Musculoskeletal adaptations and injuries due to overtraining. Exerc Sport Sci Rev, 20:99-126, 1992.

[3] Lee JM, Kim Y, Welk GJ, Validity of Consumer-Based Physical Activity Monitors. Medicine and Science in Sports and Exercise, 2014. DOI 10.1249/MSS.0000000000000287.

[4] Brage, S., Brage, N., Franks, P. W., Ekelund, U., \& Wareham, N. J. (2005). Reliability and validity of the combined heart rate and movement sensor Actiheart. European journal of clinical nutrition, 59(4), 561-570.

[5] Engström, E., Ottosson, E., Wohlfart, B., Grundström, N., \& Wisén, A. (2012). Comparison of heart rate measured by Polar RS400 and ECG, validity and repeatability. Advances in Physiotherapy, 14(3), 115-122.

[6] Goodie, J. L., Larkin, K. T., \& Schauss, S. (2000). Validation of Polar heart rate monitor for assessing heart rate during physical and mental stress. Journal of Psychophysiology, 14(3), 159.

[7] Laukkanen, R. M., \& Virtanen, P. K. (1998). Heart rate monitors: state of the art. Journal of sports sciences, 16(sup1), 3-7.

[8] Lee, C. M., \& Gorelick, M. (2011). Validity of the Smarthealth watch to measure heart rate during rest and exercise. Measurement in Physical Education and Exercise Science, 15(1), 18-25.

[9] Vanderlei, L. C. M., Silva, R. A., Pastre, C. M., Azevedo, F. M. D., \& Godoy, M. F. (2008). Comparison of the Polar S810i monitor and the ECG for the analysis of heart rate variability in the time and frequency domains. Brazilian Journal of Medical and Biological Research, 41(10), 854-859. 
[10] Maupin, G. M. (2012). Analysis of medical events among Battlefield Airmen trainees. Final Technical Report. Wright Patterson Air Force Base, AFRL-SA-WP-SR-2012-0004.

[11] Webber, B. J., Casa, D. J., Beutler, A. I., Nye, N. S., Trueblood, W. E., \& O'Connor, F. G. (2016). Preventing exertional death in military trainees: Recommendations and treatment algorithms from a multidisciplinary working group. Military Medicine, 181(4), 311-318. 